\title{
V. GABREL
}

\section{Planification à moyen terme de la production d'un satellite défilant et ensembles stables}

Revue française d'automatique, d'informatique et de recherche opérationnelle. Recherche opérationnelle, tome 29, n 2 (1995), p. 155-178.

$<$ http://www.numdam.org/item?id=RO_1995_29_2_155_0>

(C) AFCET, 1995, tous droits réservés.

L'accès aux archives de la revue « Revue française d'automatique, d'informatique et de recherche opérationnelle. Recherche opérationnelle » implique l'accord avec les conditions générales d'utilisation (http://www.numdam.org/ legal.php). Toute utilisation commerciale ou impression systématique est constitutive d'une infraction pénale. Toute copie ou impression de ce fichier doit contenir la présente mention de copyright.

\section{Numdam}

Article numérisé dans le cadre du programme

Numérisation de documents anciens mathématiques

http://www.numdam.org/ 
Recherche opérationnelle/Operations Research (vol. 29, n², 1995, p. 155 à 178)

\title{
PLANIFICATION À MOYEN TERME DE LA PRODUCTION D'UN SATELLITE DÉFILANT ET ENSEMBLES STABLES $\left({ }^{\star}\right)$
}

\author{
par V. Gabrel $\left({ }^{1}\right)$
}

Communiqué par Bernard RoY

\begin{abstract}
Résumé. - Cet article porte sur le problème combinatoire de la planification à moyen terme des images à réaliser par un satellite défilant d'observation de la Terre. Une nouvelle modélisation et des algorithmes originaux, basés sur la théorie des graphes, sont proposés; les concepts d'ensembles stables et de partitions en cliques y sont centraux. Ils permettent d'évaluer le nombre maximal d'images réalisables, d'élaborer des plans de prises de vue réalisables et d'identifier les groupes d'image qu'il est impossible de réaliser dans leur totalité. Les expérimentations numériques effectuées confirment l'efficacité de notre approche.
\end{abstract}

Mots clés : Planification, satellite d'observation de la Terre, ensemble stable, partition en cliques, graphe de comparabilité.

Abstract. - This paper deals with the combinatorial problem of medium-term production planning of shots for a low earth orbit satellite. New model and algorithms based on graph theory are proposed, they are mainly based on the notions of stable sets and partition into cliques. They aim at evaluating the maximum feasible number of images asked for, at elaborating feasible shot plans and at identifying subgroups of images that cannot be wholly taken. Numerical experiments confirm the efficiency of the proposed approach.

Keywords: Planning, Earth observing satellite, stable set, partition into cliques, comparability graph.

\section{INTRODUCTION}

Un satellite défilant d'observation de la Terre produit des images afin de satisfaire les demandes de ses utilisateurs (scientifiques, gouvernements, industriels...). En général, la quantité d'images demandées dépasse globalement les capacités de production du système. De ce fait, planifier

(*) Reçu en mars 1994.

(1) LAMSADE, Université Paris-Dauphine, place du Maréchal-de-Lattre-de-Tassigny, 75775 Paris Cedex 16, France. 
la production d'un satellite revient à la fois à sélectionner les images à réaliser et à organiser leur réalisation dans le temps en programmant les prises de vue à exécuter.

La production des satellites existants est en général planifiée d'un jour sur l'autre. Or ce type de gestion ne permet pas d'optimiser globalement l'utilisation des capacités de production. C'est en planifiant la production des images sur un horizon de temps plus long (de plusieurs jours à plusieurs semaines) qu'il est possible d'arbitrer dans le temps la réalisation des images et donc d'aboutir à une meilleure utilisation du satellite.

Des études ont été effectuées sur le problème de la planification à moyen terme de la production d'un satellite d'observation [Fab 92, Tes 88, Ver 88, Ver 89(1), Ver 89(2)]. Elles se sont attachées principalement à définir des méthodes permettant de générer un ou plusieurs plans de production à moyen terme. Cependant, compte tenu des composantes aléatoires (phénomènes météorologiques, évolution dynamique des besoins...), il s'avère souvent impossible de suivre un plan de réalisation des images à moyen terme de façon efficace tout au long de sa période d'application. Dans ce cadre, l'intérêt de la planification à moyen terme n'est pas lié à sa fonction d'ordonnancement dans le temps des tâches à réaliser, mais plutôt aux moyens qu'elle pourrait offrir pour orienter les décisions de production à court terme (d'un jour sur l'autre) afin d'éviter de décider de façon aveugle chaque jour.

C'est dans cette optique que nous proposons une méthode d'analyse de la production à moyen terme basée sur une modélisation originale, utilisant les concepts de la théorie des graphes. Cette dernière nous a amené à formuler le problème de l'élaboration de plans de réalisation des images à moyen terme comme un problème de recherche d'ensembles stables dans un graphe particulier, appelé graphe d'incompatibilité.

Afin d'évaluer la faisabilité de la charge de travail d'un satellite, nous devons déterminer le nombre de stabilité de ce graphe. Nous proposons une méthode pour borner ce nombre de stabilité : elle consiste, d'une part à déterminer une partition en cliques des sommets du graphe d'incompatibilité (nous proposons pour cela un algorithme approché exploitant les propriétés remarquables du graphe), et d'autre part à exhiber un ensemble stable maximal à partir de la partition en cliques. La partition en cliques permet également d'analyser les capacités du satellite à satisfaire les besoins des utilisateurs à moyen terme et à court terme. 
Après avoir décrit, dans la section 1 , les problèmes se posant en phase de planification à moyen terme des images à réaliser par un satellite défilant, nous mettons en valeur, dans la section 2, la non-adéquation des méthodes classiques pour les traiter. Nous montrons alors que ces problèmes sont NP-Complets. Puis, nous proposons notre modélisation; elle engendre la définition de graphes dont le graphe d'incompatibilité. En section 4, nous montrons que les propriétés remarquables du modèle permettent de simplifier la recherche d'ensembles stables maxima et l'évaluation du nombre de stabilité du graphe d'incompatibilité. Nous présentons alors les outils algorithmiques permettant de résoudre de façon approchée ces deux problèmes, à savoir l'algorithme de partition en cliques des sommets du graphe d'incompatibilité ainsi qu'un algorithme approché de détermination d'ensembles stables maximaux. Puis, nous décrivons, dans la section 5, la valeur informative d'une partition en cliques dans l'orientation des décisions de production à court terme et évoquons les recommandations qu'il est possible d'offrir sur la base de ces informations. Pour finir, nous présentons les résultats des expérimentations numériques effectuées : elles mettent en valeur la capacité des algorithmes proposés à résoudre les problèmes se posant en phase de planification à moyen terme.

\section{LE PROBLÈME DE LA GESTION DES IMAGES À RÉALISER PAR UN SATELLITE D'OBSERVATION DE LA TERRE}

\subsection{La mission d'un système d'observation de la Terre par satellite}

Un système d'observation de la Terre par satellite est conçu pour répondre aux besoins d'observation de la Terre de scientifiques, d'entreprises et/ou de gouvernements impliqués dans sa conception.

Lorsqu'un utilisateur émet une demande d'observation, il exprime ses besoins en précisant l'étendue géographique à photographier, les dates au plus tôt et au plus tard de réalisation de l'image (ce délai, appelé période de validité de la demande, peut être d'un jour, d'une semaine, de plusieurs mois, voire de plusieurs années), les exigences relatives à la qualité de l'image, à savoir la résolution, le contraste, la luminosité, la précision de localisation, et la couverture nuageuse maximale autorisée sur l'image. Satisfaire un demandeur revient à réaliser sa demande d'observation dans les conditions techniques et opérationnelles qu'il a initialement exigées. 
Les besoins d'observation des utilisateurs sont traduits en demande d'images $\left({ }^{2}\right)$, elles-mêmes traduites en prises de vue à réaliser. L'utilisation des moyens spatiaux est alors planifiée, les prises de vue programmées son exécutées, puis les images produites sont transmises au sol et traitées pour finalement être fournies sous une forme exploitable à leurs demandeurs respectifs.

Nous nous intéressons essentiellement à la phase d'élaboration de planning prévisionnel qui consiste à organiser la réalisation d'un ensemble d'images afin de satisfaire au mieux une partie ou la totalité des besoins des utilisateurs.

Dans la mesure où les besoins en matière d'observation de la Terre par satellite ont tendance à s'intensifier, les capacités productives des systèmes d'observation sont insuffisantes pour satisfaire la totalité des demandes d'image pendant leur période de validité. Ainsi, l'activité de planification de la production doit s'attacher non seulement à ordonnancer au mieux la réalisation des différentes images mais aussi à arbitrer les conflits portant sur l'utilisation des ressources.

\subsection{Description du système de production : la composante spatiale}

Le système considéré est constitué d'un satellite défilant de type SPOT1 (ce satellite particulier nous servira de référence). Un satellite défilant tourne autour de la Terre à vitesse constante en passant approximativement audessus des pôles. De par la rotation de la Terre, il survole l'intégralité de la surface terrestre au cours de son cycle orbital (à savoir l'ensemble des tours de Terre distincts réalisés). Le cycle orbital du satellite considéré est périodique, c'est-à-dire que les révolutions qu'il réalise, appelées orbites, se répètent à intervalle de temps constant. Les traces des orbites s'intersectent en deux points distincts situés approximativement à la verticale des pôles. Lorsqu'il décrit une orbite, le satellite survole pendant une demi-orbite la surface éclairée par le soleil (il va alors du pôle Sud vers le pôle Nord), et pendant l'autre demi-orbite la surface non éclairée.

Le satellite SPOT1 décrit chaque jour $(14+5 / 26)$ orbites strictement identiques à celles réalisées 26 jours auparavant. Au cours de son cycle orbital de 26 jours, il décrit 369 orbites distinctes. Pour réaliser un tour

$\left({ }^{2}\right)$ Dans la suite, nous supposerons que chaque demande d'observation peut être satisfaite en réalisant une unique image-satellite. En réalité, il est possible que la satisfaction d'une demande d'observation nécessite la réalisation de plusieurs images distinctes à des périodes différentes. Pour planifier la réalisation de ce type de demandes, nous utilisons des méthodes particulières que nous ne présenterons pas ici. 
complet du globe, ce satellite met 101 minutes. La distance maximale (à l'équateur) entre les traces de deux orbites consécutives est de $2824 \mathrm{~km}$ et la distance maximale entre les traces les plus proches de deux orbites d'un cycle est de $108 \mathrm{~km}$.

L'appareil de prise de vue embarqué à bord du satellite considéré opère dans le visible : il peut photographier la Terre uniquement de jour et ne «voit » pas à travers les nuages. Par conséquent, seules les demi-orbites de jour sont utiles pour réaliser des prises de vue. L'appareil de prise de vue considéré photographie des bandes plus ou moins longues. La prise de vue est fabriquée ligne par ligne grâce au défilement du satellite. Pour photographier un point particulier de la Terre, le satellite considéré doit se trouver sur la même latitude que ce point. Sur SPOT1, l'appareil de prise de vue embarqué peut photographier des bandes au sol de $60 \mathrm{~km}$ de large au plus. Une prise de vue peut durer de 10 secondes à plusieurs minutes.

De plus, l'appareil considéré est muni d'un miroir basculant qui lui permet de photographier à un instant donné tout point de la Terre se situant sur un segment de droite perpendiculaire à la trace du satellite. Ainsi, il peut photographier un même point de la Terre à partir de plusieurs orbites distinctes du cycle en dépassant latéralement sa direction de visée.

Les autres instruments embarqués à bord du type d'un satellite défilant sont les panneaux solaires fournissant l'énergie électrique, le (ou les) enregistreur(s) stockant les prises de vue avant de les transmettre au sol et les instruments de transmission au sol des prises de vue. Le satellite considéré est dimensionné de façon à ne pas être limité en puissance énergétique, en capacité de stockage et de transmission (ce qui est quasiment vérifié pour SPOT1).

Dans ce contexte, seule la capacité de production de l'appareil de prises de vue considéré contraint la réalisation des images. A tout instant, il peut soit effectuer une et une seule prise de vue, soit ne rien exécuter, soit effectuer certaines opérations pour passer d'un état de fonctionnement nécessaire à la réalisation d'une prise de vue particulière à un autre état adapté à la réalisation d'une prochaine prise de vue. Il est également possible que le nombre de prises de vue réalisables par un appareil de prises de vue pendant une orbite, et pendant $t$ orbites consécutives, soit limité. Concernant SPOT1, il s'avère que ce dernier type de contraintes (il s'agit de contraintes cumulatives $c f$. [Car 88]) est globalement dominé par les contraintes de type disjonctive (cf. [Car 88]) précédemment mentionnées. 


\subsection{L'élaboration de plans prévisionnels de prises de vue}

Réaliser une image revient à exécuter trois types de tâches : les prises de vue, les tâches de stockage et celles de transmission au sol des images. La planification des tâches de stockage et de transmission au sol des images ne sera pas étudiée car elle ne présente pas de difficulté particulière. En effet, les tâches de stockage sont simultanées aux prises de vue et les tâches de transmission peuvent être majoritairement réalisées pendant les demi-orbites « de nuit ».

Une prise de vue permettant de satisfaire une demande d'image est effectuée pendant un créneau d'orbite (un créneau d'orbite est exactement un créneau horaire); ainsi, toute prise de vue correspond à un couple (image, créneau d'orbite) et réciproquement. De part les capacités de déplacement de la direction de visée du satellite considéré, à une même demande d'image peut être associées plusieurs prises de vue (effectuées sur des orbites distinctes) réalisables pendant sa durée de validité $\left({ }^{3}\right)$.

Planifier la réalisation d'un ensemble de demandes d'image sur un horizon de temps donné consiste à élaborer un plan de prises de vue réalisable, c'est-à-dire à affecter à toute image au moins un créneau d'orbite (inclus dans l'horizon de planification) de façon à ne jamais affecter à un même créneau plus d'une image.

L'élaboration de plans précisionnels de prise de vue se heurte à des difficultés importantes. D'une part, la combinatoire est très élevée. S'il existe $m$ images à réaliser, le nombre de plans de prises de vue possibles est égale à :

$\prod_{i=1}^{m} \omega_{i}, \omega_{i}$ étant le nombre de prises de vue permettant de réaliser l'image $i$.

D'autre part, l'évolution du contexte de production $\left({ }^{4}\right)$ est continuelle et très difficilement prévisible : à tout moment, de nouvelles demandes peuvent être déposées, des dysfonctionnements du système productif peuvent avoir lieu, etc. A ces sources d'incertitude, classiques dans tout problème de gestion

$\left.{ }^{3}\right)$ Pour SPOT, le nombre moyen de demandes d'images en attente de traitement est de l'ordre de 5000 environ. Sur un cycle de 26 jours, le nombre d'orbites à partir desquelles il est possible de réaliser une même image varie de 1 à 9 , en fonction des exigences du demandeur, lorsque la prise de vue est situé aux alentours de l'équateur, de 1 à 11 si elle est située à $45^{\circ}$ de laltitude et, de 1 à 369 si elle est située au pôle.

$\left({ }^{4}\right)$ On entend par contexte de production l'état des moyens de production, des besoins et des conditions environnementales à un instant donné. 
de production, s'ajoute une incertitude très forte concernant la faisabilité même des images. En effet, la présence de nuages lors d'une prise de vue rend l'image obtenue inutile dès que la couverture nuageuse sur l'image dépasse celle autorisée par le demandeur.

Ces phénomènes aléatoires ont pour effet de rendre vaine l'application efficace d'un plan de prises de vue portant sur une période supérieure à la journée. Ainsi, la planification de la production des systèmes existants est le plus souvent réalisée d'un jour sur l'autre. En effet, lorsqu'on planifie les images à réaliser un jour $t$ pour le lendemain, les sources d'incertitude sont réduites à leur minimum : on connaît l'ensemble des demandes d'image à satisfaire, et on dispose de prévisions météorologiques qui, même si elles sont imparfaites, permettent d'apprécier la faisabilité de certaines images.

Or, construire chaque jour un plan de production pour le lendemain, sans considérer ce qui pourra être réalisé au-delà de ce jour, revient à découper artificiellement le problème global en sous-problèmes abusivement considérés comme indépendants : chaque jour, on choisit les prises de vue à réaliser sans savoir si, pour réaliser certaines images, il n'aurait pas été plus judicieux d'effectuer d'autres prises de vue quelques jours plus tard. Cette décomposition artificielle a pour conséquence d'éliminer le principal degré de liberté dans la planification des images à réaliser : l'arbitrage dans le temps, c'est-à-dire portant sur les orbites à partir desquelles sont effectuées les prises de vue des régions à photographier.

Il est donc utile d'insérer dans le processus de planification une phase de planification à moyen terme (portant sur plusieurs jours consécutifs voire sur plusieurs semaines) pour assurer une optimisation plus globale de la production du satellite. Son rôle ne peut être d'élaborer un calendrier d'exécution des images (on l'effectue à court terme), mais doit être d'identifier et de raisonner globalement les conflits de réalisation entre les images demandées afin de guider les choix de production à court terme. Le raisonnement à mener à moyen terme ne nécessite pas de modéliser de façon fine toutes les contraintes d'utilisation du satellite; aussi, nous ne considérerons que les contraintes dominantes à savoir les contraintes disjonctives d'utilisation de l'appareil de prises de vue.

Nous nous sommes attachés à proposer une modélisation et des outils algorithmiques pour mener à bien cette phase de planification à moyen terme, c'est-à-dire pour apporter des réponses aux questions suivantes :

- Est-il possible d'exécuter l'ensemble des demandes d'images ? En combien de temps? 
- A quels moments est-il préférable de réaliser certaines images plutôt que d'autres étant donné les conflits de réalisation et les conditions météorologiques?

- Quelle sera la quantité probable d'images distinctes réalisées pendant la période considérée?

- Quelles sont les images qu'il est avantageux de réaliser à court terme ?

2. MODÈLES ET MÉTHODES CLASSIQUES POUR PLANIFIER À MOYEN TERME LA PRODUCTION D'UN SATELLITE DÉFILANT

\subsection{Une méthode sérielle pour élaborer des plans de prises de vue à moyen terme}

Le CERT [Tes 88 , Ver 88 , Ver 89(1), Ver 89(2)] a travaillé sur le problème de la planification à moyen terme des images à réaliser par SPOT1. Son objectif d'offrir aux gestionnaires de SPOT1 un outil, utilisé en amont de l'outil de planification quotidienne, leur permettant de mieux appréhender la faisabilité globale des images à réaliser, ainsi que la faisabilité individuelle de chacune des images nouvellement demandées. Ils ont proposé une méthode appelée OSCAR, de type sérielle, pour élaborer des plans de prises de vue à moyen terme. OSCAR élabore un plan de prises de vue de façon séquentielle : les créneaux d'orbites sont affectés à la réalisation des images considérées les unes à la suite des autres. Des heuristiques sont utilisées pour définir l'ordre d'insertion des images dans le plan courant et pour, à une itération donnée, réviser les affectations précédemment établies. A chaque fois qu'une nouvelle image est demandée, son insertion peut être tentée dans le plan courant.

OSCAR répond donc à une partie des besoins qui avaient motivés sa réalisation : elle permet d'offrir une vision globale du problème de la réalisation à moyen terme d'un ensemble d'images demandées en définissant un plan de prises de vue, et elle permet de planifier «en temps réel » la réalisation des images nouvellement demandées.

Cependant, les réponses qu'elle permet d'offrir nous semblent insatisfaisantes sur trois points. Premièrement, OSCAR n'apporte aucune garantie sur la qualité des plans de prises de vue qu'il définit. Ainsi, on ne sait pas dans quelle mesure un plan de prises de vue proposé inclut ou non un nombre maximal (ou proche du maximum) de prises de vue. Deuxièmement, l'analyse de la faisabilité des images nouvellement demandées ne nous paraît pas satisfaisante car c'est dans le cadre d'un plan de prises de vue en partie 
figé qu'elle est effectuée. OSCAR ne permet pas d'appréhender de façon exhaustive les impossibilités de réalisation des demandes d'image entre elles. Troisièmement, OSCAR a implicitement été pensé dans un cadre où toutes les demandes d'image peuvent être réalisées par le satellite (avec OSCAR on cherche à savoir quand elles peuvent l'être). Or, l'accroissement des besoins en matière d'images satellitaires engendre des situations où seule une partie des besoins peut être satisfaite. Dès lors, les méthodes de planification à moyen terme doivent aider à sélectionner les images à réaliser au lieu de se polariser sur la définition d'un calendrier d'exécution de ces dernières.

\subsection{Le problème de la planification à moyen terme de la production d'un satellite défilant et les méthodes d'ordonnancement}

Pour aborder le problème de la planification à moyen terme de la production d'un satellite défilant, il est naturel de chercher à le situer dans le cadre des nombreux travaux qu'ont suscités les problèmes d'ordonnancement de fabrication ( $c f$. [Car 88, Got 93]).

Il est possible de considérer qu'il s'agit d'ordonner sur $k$ machines la réalisation de tâches indépendantes non interruptibles dont les dates de début et de fin de réalisation sont fixées, sachant qu'une machine peut exécuter au plus une tâche à chaque instant. Les tâches sont les images à réaliser, les machines sont les orbites, décrites par le satellite pendant l'horizon de planification considéré, à partir desquelles il est possible de produire les images demandées. La date de début (respectivement de fin) de réalisation d'une image $i$ est égale à la durée nécessaire au satellite pour aller du point (proche du pôle Sud) où les traces des orbites s'intersectent jusqu'à la latitude sur laquelle est située le premier (respectivement le dernier) point à photographier pour produire $i$.

Les méthodes proposées pour résoudre ce type de problèmes d'ordonnancement [Ark 87, Kol 93] ont toutes pour objectif d'élaborer un calendrier d'exécution des tâches maximisant un critère de temps ou de coût. Il s'avère que de tels critères sont absents de notre processus de décision. De plus, comme nous l'avons montré au cours des sections précédentes, définir un ou plusieurs calendriers d'exécution des images ne nous donne pas les moyens de faire jouer à la planification à moyen terme le rôle souhaité.

Pour ces raisons et pour quelques autres, nous n'avons pas pu tirer grand profit des études précédemment effectuées sur les problèmes d'ordonnancement de tâches ayant des dates de réalisation fixées. 


\subsection{La complexité des problèmes à résoudre en phase de planification à moyen terme de la production d'un satellite défilant}

Arkin et Silverberg ont prouvé dans [Ark 87] que le problème de décision (1) suivant : est-il possible de réaliser $m$ tâches sur $k$ machines non identiques $\left({ }^{5}\right)$ sachant que les dates de début et fin de réalisation des tâches sont fixées et qu'une machine peut exécuter au plus une tâche à chaque instant ? est un problème NP-Complet.

Considérons alors le problème de décision (2) se posant en phase de planification à moyen terme : est-il possible de réaliser $m$ demandes d'image sur $k$ orbites ? Les problèmes de décisions (1) et (2) sont équivalents, à une contrainte près, dans la mesure où dans le problème (2) les orbites, assimilées aux machines du problème (1), ne sont pas identiques : seul un sous-ensemble particulier d'images peut être réalisé à partir de chacune d'elles. Il existe cependant une contrainte qui distingue le problème (1) du problème (2). Dans le problème (1), un ordonnancement des tâches sur les $k$ machines est réalisable si quelles que soient deux tâches $i$ et $j$ fabriquées sur une même machine, et telles que $s_{i}<s_{j}$, on a $e_{i} \leq s_{j}, s_{i}$ (respectivement $e_{i}$ ) désignant la date de début (respectivement de fin) de $i$. Dans le problème (2), cette contrainte est quelque peu modifiée puisque on doit avoir $e_{i}+a_{i j} \leq s_{j}$, où $a_{i j}$ désigne le temps nécessaire au satellite pour réaliser $j$ à la suite de $i$. Comme $\forall i, j, a_{i j} \geq 0$, cette constante ne modifie en rien la structure et donc la complexité du problème (2) par rapport à celle du problème (1). Par conséquent, analyser la faisabilité d'un ensemble particulier d'images pendant une période de temps donnée ou de façon équivalente, élaborer des plans de prises de vue à moyen terme permettant de réaliser un certain nombre d'images est un problème NP-Complet.

3. MODÉLISATION ET FORMULATION DES PROBLÈMES À RÉSOUDRE EN PHASE DE PLANIFICATION À MOYEN TERME

\subsection{Présentation du modèle}

On considère $D$ l'ensemble des images à réaliser $(|D|=m)$ pendant l'horizon de temps considéré. Le satellite décrit $t$ orbites pendant cet horizon de planification.

$\left({ }^{5}\right)$ Les machines ne sont pas identiques dès lors que chacune d'elles ne peut exécuter qu'un sous-ensemble particulier de tâches. 
A chaque demande d'image $i$ (pour $i$ allant de 1 à $m$ ) est asocié un ensemble de prises de vue, au plus une par orbite. Quelle que soit la prise de vue considérée, on connait sa date de début de réalisation et sa durée; rappelons que ces données dépendent du défilement du satellite qui, pour réaliser la photographie d'un point donné de la Terre, doit se trouver sur la même laltitude que ce point. Toutes les prises de vue peuvent donc être ordonnées en fonction de leur date de réalisation, et on admettra par la suite que quelle que soient deux prises de vue, notées respectivement $p_{i}$ et $p_{j}$, $i<j$ implique que $t_{i}<t_{j}$ ( $t_{i}$ étant la date de début de réalisation de $p_{i}$ ). On note par $R$ l'ensemble des prises de vue possibles pour réaliser toutes les demandes d'image :

$R=\bigcup_{i \in D} \Omega_{i}$ où $\Omega_{i}$ est l'ensemble des prises de vue permettant de réaliser l'image $i(|R|=n)$.

Il est possible de définir une relation binaire sur l'ensemble $R$, appelée la relation d'enchaînabilité. Le couple $\left(p_{i}, p_{j}\right)$ appartient à la relation d'enchainnabilité, notée $A$, si et seulement si $p_{j}$ peut être réalisée à la suite de $p_{i}$, c'est-à-dire si $t_{j} \geq t_{i}+d_{i}+a_{i j}$, où $d_{i}$ désigne la durée de réalisation de $p_{i}$, et $a_{i j}$ le temps nécessaire au satellite pour passer de la réalisation de $p_{i}$ à celle de $p_{j}$. Cette relation est irréflexive, antisymétrique et transitive.

Faisons remarquer que deux prises de vue exécutées sur deux orbites distinctes sont toujours enchaînables. En effet, le satellite effectue des prises de vue pendant une demi-orbite, puis alors qu'il survole la partie nonéclairée de la Terre (pendant la seconde partie de cette même orbite), le satellite dispose toujours de suffisamment de temps pour reconfigurer son appareil de prises de vue de façon à réaliser toute prise de vue à exécuter sur l'orbite suivante, et a fortiori sur toutes les orbites successives.

Il est possible de construire un graphe orienté d'ordre $n$, noté $G_{a}=\left[X, U_{a}\right]$, dont les sommets représentent toutes les prises de vue appartenant à $R$, et dont les arcs sont définis à partir de la relation d'enchaînabilité : $\forall i, j \in X,(i j) \in U_{a} \Leftrightarrow p_{i} A p_{j}$.

En outre, l'ensemble de sommets, $X$, peut être partitionné en $m$ classes, notées $L_{i}$ pour $i$ allant de 1 à $m$, telles que les sommets constituant une même classe $i$ représentent toutes les prises de vue appartenant à $\Omega_{i}$.

\subsection{L'élaboration de plans de prises de vue à moyen terme par la détermination d'ensembles stables dans un graphe particulier}

Construire un plan de prises de vue à moyen terme réalisable consiste à sélectionner des prises de vue enchaînables, ou plus précisément un sous- 
ensemble $I$ de $X$ tel que $\forall i \in I$ et $\forall j \in I$, avec $i<j,(i j) \in U_{a} . I$ est donc un ensemble de sommets formant un chemin dans $G_{a}$. Considérons maintenant le graphe non orienté $G_{c}=\left[X, U_{c}\right]$ défini à partir de $G_{a}$ comme suit : $\forall i, j \in X,(i j) \in U_{c} \Leftrightarrow[(i j)$ et $(j i)] \notin U_{a}$. Dans ce graphe $G_{c}$, appelé le graphe de conflit, $I$ forme un ensemble stable ( $c f$. [Roy 70] et [Ber 70]) dans la mesure où $\forall i \in I$ et $\forall j \in I,(j i) \notin U_{c}$. Réciproquement, tout ensemble stable $I$ dans $G_{c}$ correspond à un chemin dans $G_{a}$.

Par conséquent, un plan de prises de vue à moyen terme réalisable correspond à un ensemble stable dans $G_{c}$. Le contraire n'est pas toujours vérifié : un ensemble stable dans $G_{c}$ ne représente pas forcément un plan de prises de vue réalisable dans la mesure où toutes les contraintes d'utilisation de l'appareil de prises de vue ne sont pas prises en compte dans notre modèle. Un plan à moyen terme défini à partir d'un ensemble stable dans $G_{c}$ ne prétend pas être un plan d'exécution effectif, il constitue un cadre pour étudier des réalisations d'images.

Un plan de prises de vue contenant au plus une prise de vue par image à réaliser, correspond nécessairement à un ensemble, noté $S$, tel que $S$ est un ensemble stable dans $G_{c}$ et $\forall i \in\{1, \ldots, m\},\left|S \cap L_{i}\right| \leq 1$. Par conséquent, $S$ est un ensemble stable dans le graphe, noté $G=[X, U]$ et appelé le graphe d'incompatibilité, dont l'ensemble des arêtes est défini comme suit : $\forall i, j \in X,(i j) \in U \Leftrightarrow\left[(i j) \in U_{c}\right.$ ou $\left.\exists k \in\{1, \ldots, m\} /(i, j) \in L_{k} \times L_{k}\right]$.

Ce graphe d'incompatibilité modélise deux relations binaires sur $R \times R$ : la relation de conflit et la relation d'exclusion. La relation de conflit est donc la relation complémentaire de la relation d'enchaînabilité transformée en relation symétrique, cette relation est irréflexive, symétrique et antitransitive. Et, la relation d'exclusion inclut toute paire de prises de vue permettant de réaliser une même demande d'image, cette relation est irréflexive, symétrique et transitive. Sur la base de la relation d'exclusion est défini un dernier graphe non orienté, appelé le graphe d'exclusion et noté $G_{e}=\left[X, U_{e}\right]$. Faisons remarquer que les prises de vue appartenant à une même clas'se $L_{i}$ sont représentées par des sommets formant une clique dans $G_{e}$. Ainsi, $G_{e}$ est par constitution une collection de disques disjointes.

Le nombre d'images programmées dans un plan de prises de vue représenté par $S$ est égal à la cardinalité de $S$. Ainsi, un plan de prises de vue qui maximise le nombre d'images programmées en sélectionnant au plus une prise de vue par image, correspond nécessairement à un ensemble stable maximum dans $G$. 
Par conséquent, il n'est possible de réaliser toutes les demandes d'image sur les $t$ orbites considérées que s'il existe un ensemble stable dans $G$ de cardinalité égal au nombre d'images demandées. S'il en est ainsi, pourvu que les contraintes de production négligées n'entravent pas la réalisabilité du plan, il sera effectivement possible d'exécuter toutes les demandes d'image. Sinon, il est certain que toutes les demandes d'image ne pourront être exécutées.

Déterminer le nombre de stabilité (ce nombre est le cardinal d'un ensemble stable maximum) de $G$ permet donc d'évaluer la capacité du système à satisfaire les $m$ demandes d'image sur les $t$ orbites considérées. Ce problème étant NP-Complet, nous nous attachons à estimer ce nombre de stabilité en le bornant supérieurement et inférieurement.

\section{LES MÉTHODES DE RÉSOLUTION}

\subsection{Une borne supérieure évidente pour le nombre de stabilité du graphe d'incompatibilité}

Étant donné qu'un ensemble stable dans le graphe d'incompatibilité est un stable dans le graphe de conflit et dans le graphe d'exclusion, il est possible de majorer le nombre de stabilité de $G$ par le nombre de stabilité de $G_{C}$ et le nombre de stabilité de $G_{e}$ :

$$
\alpha(G) \leq \min \left(\alpha\left(G_{c}\right), \alpha\left(G_{e}\right)\right) .
$$

Il s'avère que le nombre de stabilité de $G_{c}$ et celui de $G_{e}$ peuvent être déterminés en un temps polynomial.

Par construction, le graphe d'exclusion $G_{e}$ est constitué de $m$ cliques deux à deux disjointes $\left({ }^{6}\right)$. Dans un tel graphe, un ensemble $S$, pour être stable, ne peut posséder plus d'un sommet dans chacune de ces cliques, $|S|$ est donc au plus égal à $m$. Si on choisit un sommet arbitrairement dans chacune des $m$ cliques, on obtient un ensemble stable maximum de cardinalité $m$. Le nombre de stabilité de $G_{e}$ est donc égal à $m$.

Pour étudier la cardinalité des ensembles stables maxima de $G_{C}$, il suffit de travailler sur $G_{a}$. Rappelons qu'un ensemble stable dans $G_{c}$ correspond à un chemin dans $G_{a}$ et réciproquement. Aussi, déterminer le nombre de stabilité de $G_{C}$ revient à exhiber le nombre maximal de sommets pouvant appartenir à un chemin dans $G_{a}$. La relation d'enchaînabilité étant antisymétrique et

$\left({ }^{6}\right)$ Rappelons qu'une clique maximale est formée de tous les sommets représentant les différentes prises de vue permettant de satisfaire une même demande d'image. 
transitive, $G_{a}$ est un graphe orienté sans circuit au sein duquel la recherche du plus long chemin peut s'effectuer en un temps polynomial (cf. [Gar 79]).

Majorer le nombre de stabilité de $G$ par le minimum entre le nombre de stabilité de $G_{C}$ et celui de $G_{e}$ reste assez grossier. Nous nous sommes donc attachés à affiner cette borne pour pouvoir évaluer de façon plus précise la faisabilité d'un ensemble de demandes d'image à moyen terme.

\subsection{Une méthode approchée pour majorer le nombre de stabilité de $G$}

La détermination d'une partition, notée $C$, de $X$ en cliques dans $G$ nous permet de trouver une nouvelle borne supérieure pour le nombre de stabilité de $G$ car $\alpha(G) \leq|C|$. En effet, étant donné $\theta(H)$ le plus petit nombre de cliques qui partitionnent les sommets d'un graphe quelconque $H=[V, W]$, on a $\alpha(H) \leq \theta(H)(\alpha(H)$ étant le nombre de stabilité de $H)$.

Cette approche est motivée par le fait que la notion de cliques intervient très naturellement dans notre modèle : le graphe d'exclusion est une collection de cliques disjointes. De plus, il est possible de déterminer en un temps polynomial une partition minimum en cliques de $X$ dans $G_{c}$. Pour le démontrer, faisons au préalable observer que dans un graphe quel qu'il soit, une partition minimum en cliques de ses sommets correspond dans son graphe complémentaire à une coloration minimum de ses sommets. Le graphe complémentaire de $G_{c}$ étant un graphe de comparabilité $\left(^{7}\right)$ (ce qui est évident puisque ce graphe n'est autre que la version non orientée de $G_{a}$, graphe d'une relation d'ordre), il est possible de déterminer une coloration minimum de ses sommets en un temps polynomial [Even 72].

En outre, nous avons pu exhiber des propriétés remarquables sur $G$ qui tendent à simplifier la recherche d'une partition en cliques de $X$ dans $G$ (le problème de la détermination d'une partition minimum restant NP-Complet).

Proposition 1 : Une clique quelconque de $G$ est soit une clique de $G_{e}$, soit une clique de $G_{c}$. Plusieurs cas doivent être considérés :

- Concernant les 1-cliques $\left({ }^{8}\right)$ de $G$. Ces cliques sont les sommets de $G$ qui sont également ceux de $G_{c}$ et de $G_{e}$; donc, toute 1-clique de $G$ est une 1-clique de $G_{e}$ et de $G_{c}$.

${ }^{7}$ ) Un graphe simple non orienté $H=[V, W]$ est appelé graphe de comparabilité s'il est possible en orientant ses arêtes d'en faire le graphe orienté $H^{\prime}=\left[V, W^{\prime}\right]$ d'une relation d'ordre, c'est-à-dire d'une relation antisymétrique et transitive ( $c f$. [Ber 70]).

$\left({ }^{8}\right)$ Une $i$-clique est une clique de cardinal égal à $i$. 
- Concernant les 2-cliques de G. Ces cliques sont les arêtes de $G$, or une arête de $G$ est soit une arête de $G_{e}$, soit une arête de $G_{c}$. Par conséquent, toute 2-clique dans $G$ est une 2-clique dans $G_{e}$ ou dans $G_{c}$.

- Concernant les 3-cliques de $G$. Soit un triangle $(i, j, k)$ de $G$, on distingue 2 sous-cas :

(a) $(i j) \in U_{c}$ et $(i k),(j k) \in U_{e}$.

Comme la relation d'exclusion est symétrique et transitive, si $(i k),(j k) \in U_{e}$ alors $(i j) \in U_{e}$. Dans ce cas, $(i j)$ ne peut être une arête du graphe de conflit. En effet, rappelons que deux prises de vue permettant de réaliser une même image sont nécessairement réalisées à partir de deux orbites distinctes, et que deux prises de vue réalisables sur deux orbites distinctes sont toujours enchaînables.

(b) $(i j),(i k) \in U_{c}$ et $(j k) \in U_{e}$.

Puisque $(i j) \in U_{c}$, $i$ et $j$ représentent deux prises de vue réalisables sur la même orbite, il en est de même pour $i$ et $k$. Par conséquent, $i, j$ et $k$ sont réalisables sur la même orbite, et donc $(j k)$ ne peut être une arête du graphe d'exclusion.

Les cas considérés ci-dessus sont suffisants. En effet, étant donné une $k$ clique de $G$ (avec $k>3$ ), si elle contient des arêtes de $G_{e}$ et de $G_{c}$, alors il existe nécessairement au moins un triangle contenant à la fois des arêtes de $G_{e}$ et de $G_{c}$, ce qui est impossible comme nous l'avons prouvé précédemment.

Proposition 2: Soit $P_{i}$ une clique de $G_{e}$ appartenant à une partition minimum en cliques de $X$ dans $G$ notée $P$, il est toujours possible d'ajouter des sommets dans $P_{i}$ afin qu'elle devienne maximale dans $G_{e}$ sans détruire la minimalité de $P$.

Soit $L_{j}$ une clique maximale de $G_{e}$ telle que $P_{i}$ est inclue dans $L_{j}$. Supposons sans perte de généralité que $P_{i}=L_{j} \backslash\{i, j\}$. Puisque $P$ est minimum, $(i, j)$ ne peut être une clique de $P$; par conséquent, $i$ et $j$ appartient à deux cliques distinctes formées par des arêtes de $G_{c}$, notons ces cliques $P_{k}$ et $P_{v}$. Dans ce cas, il est possible d'exclure $i$ et $j$ de $P_{k}$ et $P_{v}$ respectivement et de les ajouter dans $P_{i}$ sans changer la cardinalité de $P$.

Les deux propositions présentées précédemment permettent d'affirmer qu'il existe une partition minimum en cliques de $X$ dans $G$ constituées de cliques de $G_{c}$ et/ou de cliques maximales de $G_{e}$.

L'algorithme approché proposé pour déterminer une partition en cliques de $X$ dans $G$ se déroule comme suit. A l'initialisation, cet algorithme dispose 
de la partition minimum en cliques de $X$ dans $G_{e}$, notée $L=\left\{L_{1}, \ldots, L_{m}\right\}$. A une itération $i$, on note $V$ l'ensemble des sommets considérés, $G_{c}(V)$ (respectivement $G_{e}(V)$ ) le sous-graphe de conflit engendré par $V$ (le sousgraphe d'exclusion engendré par $V$ ) et, $L^{c}(V)(L(V))$ la partition minimum en cliques de $V$ dans $G_{C}(V)$ (la partition minimum en cliques de $V$ sur $\left.G_{e}(V)\right)$. On calcule $L^{c}(V)$ à l'aide de la procédure CLIQUES appliquée à $G_{c}(V)\left({ }^{9}\right)$. Si $L^{c}(V)$ ne contient pas de 1-cliques, cette itération est la dernière et la partition de $X$ dans $G$ retenue, notée $C$, est constituée des cliques de $L^{c}(V)$ et des cliques de $L(X \backslash V)$. Sinon, on considère une à une chacune des 1-cliques de $L^{c}(V)$. Considérant une 1-clique particulière, si le sommet qui la constitue appartient dans $L$ à une clique "d'exclusion » de taille supérieure à 1 , soit $L_{i}$ cette clique, on exclut les sommets de $L_{i}$ de l'ensemble $V$. Lorsque toutes les 1-cliques ont été traitées, si $V$ n'a pas été modifié, cette itération est la dernière et $C$ est constituée de la même façon que précédemment, sinon on passe à l'itération $(i+1)$.

A l'issue de la dernière itération, on retient comme partition de $X$ dans $G$ la partition de plus petite cardinalité entre $C$ et $L$.

Cet algorithme a une complexité dans le pire cas de $O\left(n^{3}\right)$, alors que la complexité de CLIQUES est de $O\left(n^{2}\right)$. Cet algorithme est déroulé sur un exemple simple en annexe.

On dispose donc d'une borne supérieure pour le nombre de stabilité de $G$. Pour exhiber une borne inférieure, il suffit de déterminer un ensemble stable, $S$, dans $G$. On obtient alors :

$$
|S| \leq \alpha(G) \leq|C|
$$

\subsection{Un algorithme approché pour minorer le nombre de stabilité de $G$}

Pour déterminer $S$, nous proposons un algorithme approché, utilisant $C$ la partition en cliques de $X$ dans $G$. $S$ est construit de façon gloutonne en sélectionnant 0 et 1 sommet dans chaque clique de $C$ de façon à ne jamais retenir deux sommets adjacents. A la première itération, $V$, l'ensemble des sommets pouvant appartenir à $S$, est constitué de tous les sommets de $X$. Puis, à chaque itération et tant que $V \neq \varnothing$, on ajoute dans $S$ le sommet $i$ de

$\left({ }^{9}\right)$ Cette procédure CLIQUES appliquée à un graphe $H$ permet de déterminer une partition minimum en cliques des sommets de $H$ si et seulement si $H$ est le graphe complémentaire d'un graphe de comparabilité. Pour cela, elle détermine une coloration minimum des sommets du graphe complémentaire de $H$, l'algorithme de coloration est exactement celui présenté dans [Even 72]. 
Algorithme de partition en cliques du graphe d'incompatibilité

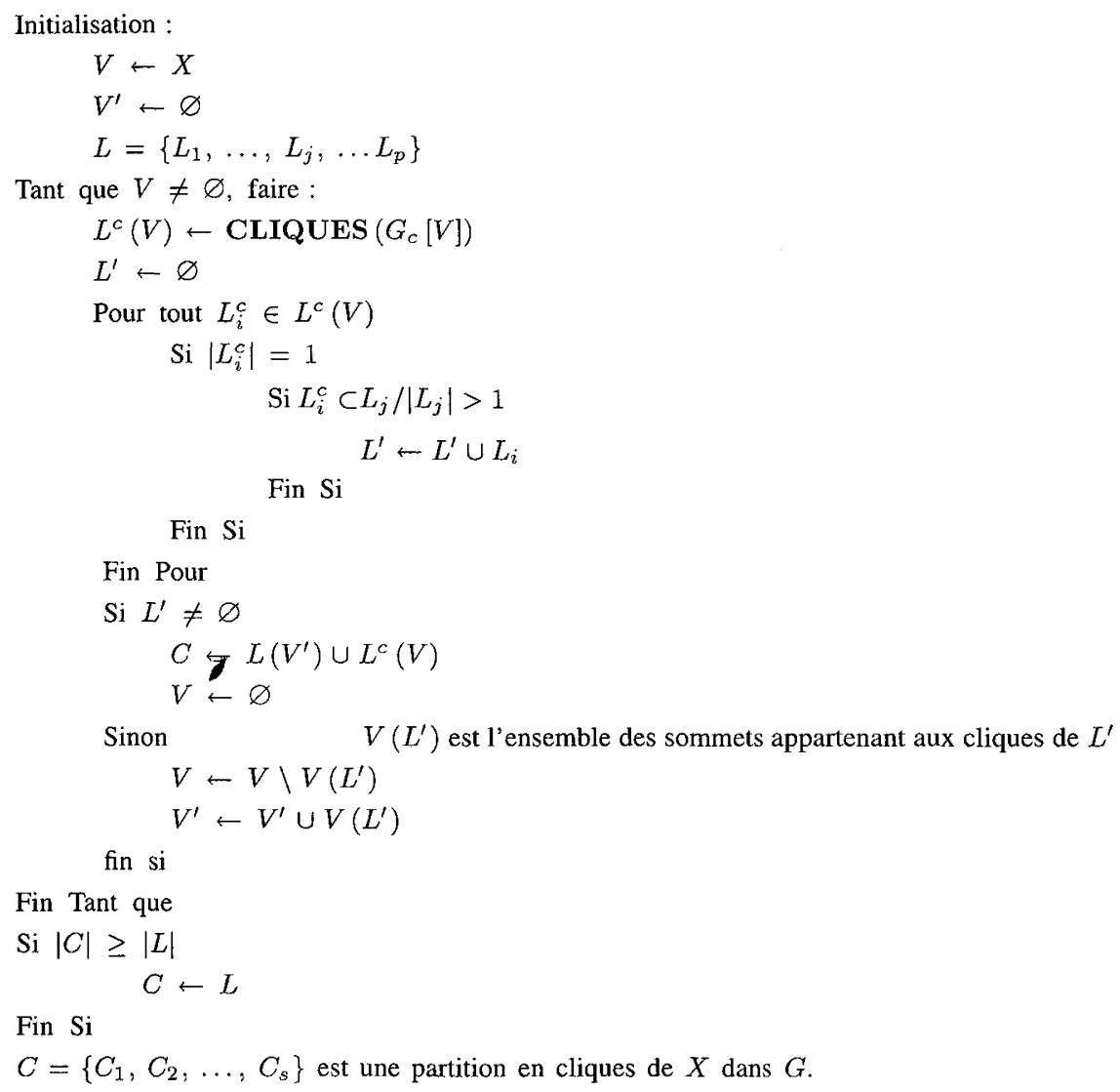

$$
\begin{aligned}
& V \leftarrow X \\
& V^{\prime} \leftarrow \varnothing \\
& L=\left\{L_{1}, \ldots, L_{j}, \ldots L_{p}\right\}
\end{aligned}
$$

Tant que $V \neq \varnothing$, faire :

$$
\begin{aligned}
& L^{c}(V) \leftarrow \text { CLIQUES }\left(G_{c}[V]\right) \\
& L^{\prime} \leftarrow \varnothing
\end{aligned}
$$$$
\text { Pour tout } L_{i}^{c} \in L^{c}(V)
$$$$
\text { Si }\left|L_{i}^{c}\right|=1
$$$$
\text { Si } L_{i}^{c} \subset L_{j} /\left|L_{j}\right|>1
$$$$
L^{\prime} \leftarrow L^{\prime} \cup L_{i}
$$

Fin $\mathrm{Si}$

Fin $\mathrm{Si}$

Fin Pour

Si $L^{\prime} \neq \varnothing$$$
\begin{aligned}
& C \nsucceq L\left(V^{\prime}\right) \cup L^{c}(V) \\
& V \leftarrow \varnothing
\end{aligned}
$$

Sinon $V\left(L^{\prime}\right)$ est l'ensemble des sommets appartenant aux cliques de $L^{\prime}$

$$
\begin{aligned}
& V \leftarrow V \backslash V\left(L^{\prime}\right) \\
& V^{\prime} \leftarrow V^{\prime} \cup V\left(L^{\prime}\right)
\end{aligned}
$$

fin si

Fin Tant que

Si $|C| \geq|L|$

$$
C \leftarrow L
$$

Fin $\mathrm{Si}$

$C=\left\{C_{1}, C_{2}, \ldots, C_{s}\right\}$ est une partition en cliques de $X$ dans $G$.

$V$ qui a, au sein de la (ou des) clique(s) de plus petite cardinalité dans $C$, le plus petit degré dans $G(V)$ (le sous-graphe de $G$ engendré par $V$ ), et on exclut de $V$ tous les sommets adjacents à $i$. On remarque que si $|S|=|C|$, $S$ est un ensemble stable maximum, $C$ une partition minimum.

Il est donc possible de borner le nombre de stabilité de $G$, et nous verrons à la section 6 que les bornes obtenues permettent de l'encadrer de façon satisfaisante. En outre, la partition en cliques de $X$ dans $G$ permet d'obtenir des informations synthétiques sur les différentes opportunités de réalisation des images demandées (considérées individuellement ou globalement) pendant l'horizon temporel considéré. 
5. L'ANALYSE MENÉE SUR LE MOYEN TERME ET SES CONSÉQUENCES SUR LES DÉCISIONS DE PRODUCTION À COURT TERME

Nous pouvons analyser les capacités de production du satellite à moyen terme en travaillant sur le graphe d'incompatibilité, ou de façon similaire, sur chaque composante connexe du graphe d'incompatibilité.

\section{1. Évaluation du nombre de demandes d'image réalisables par le satellite et de la période nécessaire pour les exécuter}

Pour savoir si $m$ demandes d'image peuvent être exécutées au moins une fois pendant leur période de validité, il faut générer un plan à moyen terme sur le plus grand horizon de planification envisageable, c'est-à-dire celui couvrant toutes les durées de validité de ces demandes d'image.

$\mathrm{Si}$, dans le graphe d'incompatibilité associé au problème de la réalisation de ces images sur cet horizon de planification, le cardinal de la partition en cliques des sommets est inférieur à $m$, toutes ces demandes d'image ne peuvent être satisfaites.

Si le cardinal de l'ensemble stable, généré par l'algorithme approché, est égal à $m$, il est possible de réaliser les $m$ demandes d'image. Et, en réduisant pas à pas l'horizon de planification, on peut évaluer la période de temps minimale pendant laquelle il est possible d'exécuter toutes ces demandes d'image.

\subsection{Les recommandations concernant les images à réaliser}

\subsubsection{Les recommandations individuelles}

En exploitant $C$, la partition en cliques de $X$ dans $G$, il est possible d'identifier les images réalisables, ainsi que celles dont la réalisation empêche l'exécution d'autres images.

Ainsi, lorsque $C$ admet une clique $L_{i}$ du graphe d'exclusion, il est fort probable de pouvoir réaliser l'image $i$ pendant l'horizon de temps considéré. En effet, rappelons que pour élaborer un ensemble stable, il suffit de sélectionner, dans chaque clique de $C, 0$ ou 1 sommet de façon à ne jamais retenir deux sommets adjacents. Par conséquent, si $L_{i}$ appartient à $C$, il est fort probable de pouvoir inclure un sommet de $L_{i}$ dans un stable maximum, et donc de pouvour exécuter l'image $i$ au moins une fois. De plus, si parmi les sommets de $L_{i}$, il existe un ou plusieurs sommets de degré 0 dans le graphe de conflit, il est certain que, toutes choses étant égales par ailleurs, l'image peut être effectuée. 
A contrario, les sommets constituant une clique appartenant à $C$ et formée par des arêtes de $G_{c}$, représentent des prises de vue permettant de réaliser des images distinctes qu'il n'est probablement pas possible de toutes exécuter. Considérons un sommet $i$ appartenant à une telle clique et permettant d'effectuer l'image $k$, il s'avère, de part la construction de $C$, que tous les sommets de $L_{k}$ appartiennent à des cliques distinctes dans $C$. De l'ensemble de ces cliques, il est possible de déduire l'ensemble des demandes d'image dont les réalisations peuvent empêcher la réalisation de l'image $\mathrm{K}$.

Pour identifier les groupes d'images qui ne pourront pas toutes être réalisées, il suffit de construire un graphe, noté $G_{I^{\prime}}=\left[Y, U_{I}\right]$ où chaque sommet représente une image à réaliser, et où il existe une arête de couleur $q$ entre deux sommets $i$ et $j(i \neq j)$ si et seulement si $\exists k \in L_{i}$ et $\exists j \in L_{j}$ : $(k, l) \in C_{q} \times C_{q}, C_{q} \in C$.

Un sommet $i$ a un degré égal à 0 dans $G_{I}$ si $L_{i}$ appartient à $C$. Hormis ces sommets isolés, les composantes connexes de $G_{I}$ sont constituées de sommets représentant des images qu'il est très probablement impossible de toutes réaliser. En fait, considérant une composante connexe particulière constituée de $v$ sommets et d'arêtes coloriées par $w$ couleurs distinctes, le nombre maximal de prises de vue qu'il est possible de réaliser afin de satisfaire les $v$ demandes d'images représentées par ces $v$ sommets est égal à $w$. En effet, lorsqu'il existe $w$ arcs de couleurs distinctes, l'ensemble des sommets représentant les prises de vue permettant de réaliser ces $v$ demandes d'image, est partitionné en $w$ cliques dans $C$, et par conséquent, il est impossible de réaliser plus de $w$ prises de vue pour exécuter ces images. Ainsi, considérant chacune des composantes connexes de $G_{I}$, si $v>w$, on sait que les $v$ demandes d'images représentées par les $v$ sommets ne peuvent toutes être satisfaites (on ne peut au mieux exécuter que $w$ d'entre elles); et, il n'est possible de réaliser l'intégralité de ces $v$ images que si $v=w$. Faisons remarquer que plus la partition en cliques de $X$ dans $G$ est proche de l'optimum, plus les informations relatives à la faisabilité des images sont pertinentes. En fait, en travaillant sur une partition sous-optimale, on sous-évalue les impossibilités de réalisation entre les images demandées.

\subsubsection{Les recommandations globales sur les choix de production à court terme}

Il est possible en utilisant la partition en cliques $C$ et des données permettant de prévoir les conditions météorologiques (des probabilités de couverture nuageuse sont disponibles sur 2 à 3 jours), de partitionner l'ensemble des images à réaliser sur une région donnée en plusieurs catégories. La première catégorie regroupe les images dont la réalisation est 
préférable à court terme. La deuxième catégorie regroupe des images dont la réalisation doit être retardée. La dernière catégorie regroupe les images pour lesquelles il est plus difficile de mesurer l'intérêt d'une réalisation à court terme.

Nous travaillons actuellement sur la définition d'une méthode d'affectation rigide et formelle des demandes d'image à l'une des trois catégories basée sur une analyse multicritère du problème. Il est envisagé d'utiliser ELECTRE TRI, une méthode de segmentation élaborée au LAMSADE par B. Roy et Y. Wei [Roy 93], en prenant en considération les critères d'évaluation d'une demande d'image suivants : les conditions météorologiques de réalisation à court terme (on utilise les prévisions de couverture nuageuse), les conditions météorologiques de réalisation à moyen terme (on utilise les données climatiques), les impossibilités de réalisation entre demandes d'image, le nombre de prises de vue en conflit avec la prise de vue à court terme associée à la demande d'image considérée, le nombre total de prises de vue permettant de réaliser l'image.

\section{EXPÉRIMENTATIONS NUMÉRIQUES}

Nous avons généré 700 instances distinctes. Pour construire les 400 premières instances, nous positionnons aléatoirement $m(m=\{50,100,200$, 300 \}) demandes d'image dans une région, située à l'équateur, de $2000 \mathrm{~km}$ de large sur $3000 \mathrm{~km}$ de long; nous considérons que ces images peuvent être réalisées pendant les 26 jours à venir (durant cette période le satellite décrit 27 orbites en vue de la région), l'angle de roulis autorisé pour réaliser chacune de ces demandes d'image est choisi aléatoirement entre $5,10,15$, 20,25 et $30^{\circ}$, et la durée de toute prise de vue permettant de réaliser chacune de ces images est fixée à 10 secondes (il est en effet inutile de faire varier la durée des prises de vue dans la mesure où cette dernière n'est pas envisagée dans nos expérimentations comme un paramètre à optimiser). A partir de ces données, il est possible de construire les 400 graphes d'incompatibilité représentatifs de ces 400 instances. Pour définir les 300 dernières instances, nous avons reconsidéré les 300 premières instances où 50,100 et 200 demandes d'image sont aléatgirement positionnées dans une région située à l'équateur, mais on place maintenant cette région à $45^{\circ}$ de latitude. A cette latitude, le nombre d'orbites décrites pendant 26 jours par le satellite est de 38 (au lieu de 27 précédemment). Aussi, lorsqu'on déplace la région de l'équateur à $45^{\circ}$ de latitude, il existe un plus grand nombre de prises de vue possibles pour réaliser chacune des images demandées, et par conséquent, ceci nous amène à définir 300 graphes d'incompatibilité supplémentaires. 
L'ordre de chacun de ces graphes d'incompatibilité varie de 194 à 1460 et vaut en moyenne 756. La densité (à savoir le nombre d'arêtes divisé par le nombre de sommets d'un graphe) moyenne de ces graphes est de 8 , la plus faible densité est de 3 alors que la plus forte est de 15,5.

Pour chaque instance, nous avons déterminé $C$ une partition en cliques de $X$ dans $G$ et $S$ un ensemble stable maximal à partir de cette partition en cliques. Les temps d'exécution de ces deux algorithmes sont très rapides: moins de 2 à 3 minutes en moyenne sur une SPARCStation2 (de 0 à 5 minutes).

Afin d'estimer la capacité des algorithmes approchés proposés à encadrer le nombre de stabilité d'un graphe d'incompatibilité, nous avons calculé pour chaque instance le rapport :

$$
\frac{|S|}{|C|}
$$

En moyenne, ce rapport est très bon puisqu'il est égal à 0,94 ; dans le pire cas, il vaut 0,82 et dans le meilleur des cas il est égal à 1 . Les résultats sont présentés de façon synthétique dans la figure ci-après.

En outre, remarquons que ce rapport est une sous-évaluation du rapport d'approximation $\left({ }^{10}\right)$ de l'algorithme approché de détermination d'un ensemble stable maximal dans $G$ :

$$
\alpha(G) \leq|C| \quad \Leftrightarrow \quad \frac{|S|}{\alpha(G)} \geq \frac{|S|}{|C|}
$$

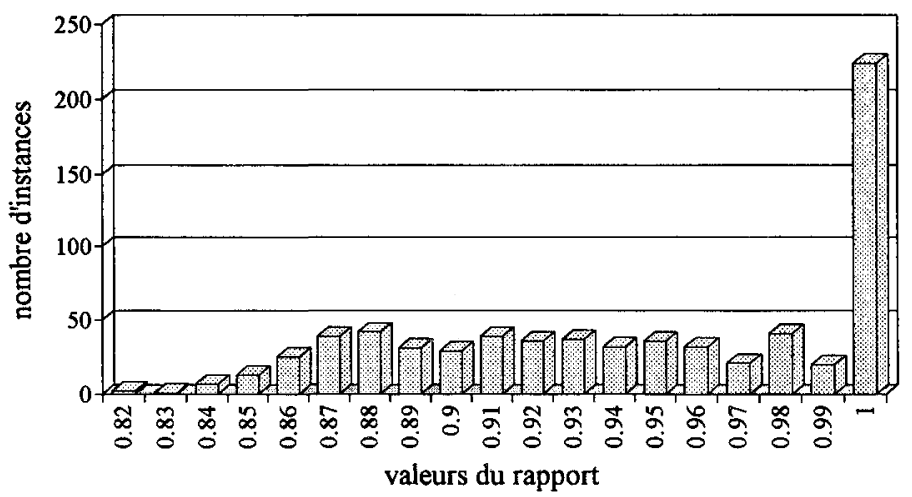

\footnotetext{
$\left({ }^{10}\right)$ Le rapport d'approximation est égal à la valeur de la solution fournie par un algorithme approché divisée par la valeur de la solution optimale.
} 
Les résultats de ces expérimentations numériques sont donc très satisfaisants : ils mettent en valeur la pertinence des algorithmes proposés pour encadrer le nombre de stabilité d'un graphe d'incompatibilité, et pour définir une partition en cliques des sommets d'un tel graphe dont la cardinalité est proche du minimum.

\section{CONCLUSION}

Contrairement aux outils de planification élaborés précédemment, la méthodologie proposée dans cet article a pour objectif d'attribuer à la planification à moyen terme un rôle distinct, voire complémentaire, de celui de la planification à court terme. Son rôle est de guider les choix de production à court terme, réalisés de façon séquentielle, afin d'approcher un « optimum global».

L'originalité de notre modélisation et les algorithmes qu'elle a permis de définir nous donnent la possibilité de mener à bien cette phase de planification à moyen terme des images à réaliser par satellite. En effet, les algorithmes proposés permettent d'estimer le nombre maximal d'images distinctes réalisables pendant un horizon de temps particulier, d'élaborer un ou des plans à moyen terme permettant de réaliser un nombre d'images proche du maximum, d'identifier les groupes d'images qu'il est possible de réaliser dans leur totalité, et de guider les décisions à court terme.

Nous disposons donc d'outils algorithmiques pour envisager la planification à moyen terme, non plus comme fonction de séquencement de tâches sur un horizon de plusieurs jours consécutifs, mais plutôt comme fonction d'orientation des décisions de production à court terme vers des objectifs globaux.

La modélisation effectuée et les algorithmes proposés l'ont été dans le cadre d'un système d'observation composé d'un unique satellite à optique visible. Nous travaillons sur une généralisation de notre étude afin de traiter les problèmes de planification à moyen terme de systèmes d'observation multi-satellites, multi-senseurs.

\section{REMERCIEMENTS}

Je tiens à remercier très chaleureusement $\mathrm{A}$. Moulet, V. Paschos et B. Roy pour le soutien et les conseils qu'ils m'ont prodigués. 


\section{RÉFÉRENCES}

[ARK 87] E. M. ARkin et E. B. Stluerberg, Scheduling jobs with fixed start and end times, Discrete Applied Mathematics, 1978, 18, p.1-8.

[BER 70] C. BERGE, Graphes et hypergraphes, Dunod, 1970.

[CAR 88] J. Carlier et P. Chrétienne, Problèmes d'ordonnancement, Masson, 1988.

[EVen 72] S. Even, A. Lempel et A. Pnuel, Permutation graphs and transitive graphs, Journal of the Association for Computing Machinery, July 1972, $19, \mathrm{n}^{\circ} 3$, p. $400-410$.

[FAB 92] P. FABIANI, Planification de mission d'un satellite d'observation, Rapport de stage de DEA, ENSAE, Toulouse, septembre 1992.

[GAR 79] M. R. GAREY et D. S. Johnson, Computers and Intractability: a guide to the theory of NP-Completeness, Freeman, San Francisco, 1979.

[GON 85] M. Gondran et M. Minoux, Graphes et Algorithmes, Eyrolles, 1985.

[GOT 93] GOTHA, Les problèmes d'ordonnancement, Recherche Opérationnelle, 1993, 27, n 1, p. 77-150.

[KOL 93] A. W. J. Kolen et L. G. KROoN, On the computational complexity of (maximum) shift class scheduling, European Journal of Operational Research, 1993, 164, p. 138-151.

[ROY 70] B. Roy, Algèbre moderne et théorie des graphes, Tome 2, Dunod, 1970.

[ROY 93] B. Roy et D. Bouyssou, Aide Multicritère à la Décision : Méthodes et Cas, Economica, 1993.

[TES 88] C. TeSSIER-BADIE, Contribution à l'étude des problèmes d'affectation de ressources et d'ordonnancement : application au domaine spatial, Thèse ENSAE, Toulouse, 1988.

[VER 88] G. VeRFAILlIE et C. BADIE, Étude en vue de développer une méthodologie et des outils opérationnels pour la planification de missions spatiales, CERT, Rapport intermédiaire $n^{\circ} 1 / 3330 / D E R I$, juin 1988, et Rapport final $n^{\circ}$ 2/3330/DERI, septembre 1988 .

[VER 89(1)] G. Verfaillie et C. Gaspin, Présentation et comparaison de deux approches $d u$ problème d'ordonnancement, CERT, Rapport final $n^{\circ} 1 / 3335 /$ DERI, septembre 1989.

[VER 89(2)] G. VeRFAILLIE, Étude en vue de développer une méthodologie et des outils opérationnels pour la planification de missions spatiales, CERT, Rapport intermédaire $n^{\circ} 1 / 3340 / D E R I$, octobre 1989 et Rapport final $n^{\circ}$ 2/3340/DERI, mars 1990 .

\section{ANNEXE}

On considère 9 prises de vue permettant de réaliser 5 demandes d'image : les prises de vue 1 et 2 permettent de réaliser $P_{1}, 3$ et 4 permettent de 
réaliser $P_{2}, 5$ et 6 sont associées à la réalisation de $P_{3}, 7$ et 8 à celle $P_{4}$ et 9 à celle $P_{5}$. Le graphe d'incompatibilité associé est le suivant :

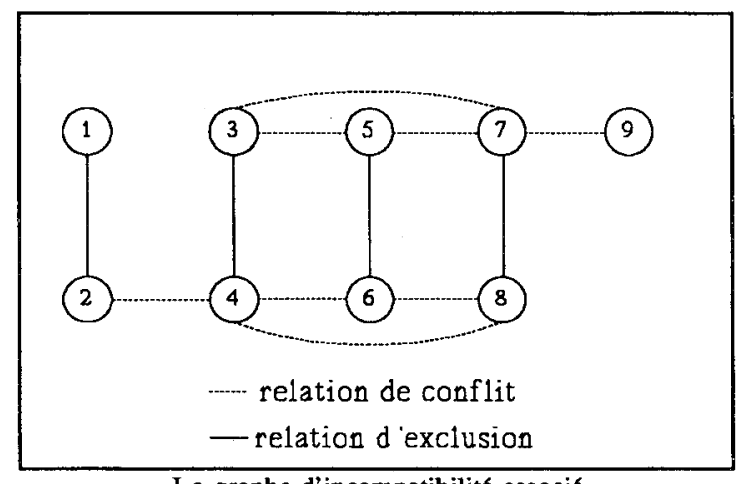

Le graphe d'incompatibilité associé

$$
\begin{aligned}
& L=\left\{L_{1}, L_{2}, L_{3}, L_{4}, L_{5}\right\} \\
& L_{1}=\{1,2\} ; L_{2}=\{3,4\} ; L_{3}=\{5,6\} ; L_{4}=\{7,8\} ; L_{5}=\{9\} . \\
& i=1: L^{c}=\left\{L_{1}^{c}, L_{2}^{c}, L_{3}^{c}, L_{4}^{c}, L_{5}^{c}\right\} \\
& L_{1}^{c}=\{1\} ; L_{2}^{c}=\{2,4\} ; L_{3}^{c}=\{3,5,7\} ; L_{4}^{c}=\{6,8\} ; L_{5}^{c}=\{9\} \\
& L^{\prime}=\{(1,2)\} \\
& i=2: L^{c}=\left\{L_{1}^{c}, L_{2}^{c}, L_{3}^{c}\right\} \\
& L_{1}^{c}=\{3,5,7\} ; L_{2}^{c}=\{4,6,8\} ; L_{3}^{c}=\{9\} \\
& L^{\prime} \neq \varnothing \\
& P=L^{c}(V) \cup L\left(V^{\prime}\right)=\{(3,5,7),(4,6,8),(9),(1,2)\} \\
& |P|<|L| \Rightarrow C=P \\
& C=\left\{C_{1}, C_{2}, C_{3}, C_{4}\right\} \text { est la partition en cliques de } X \text { sur } G \text { avec : } \\
& C_{1}=(3,5,7) ; C_{2}=(4,6,8) ; C_{3}=L_{1} ; C_{4}=L_{5}
\end{aligned}
$$

Cette partition est minimum puisqu'il est possible d'exhiber un ensemble stable de cardinalité égale à $4: S^{*}=\{1,4,5,9\}$. 